\title{
Reconstruction of the anterior chest wall with a 3-dimensionally printed biodynamic prosthesis
}

\author{
Jose Ramón Cano, MD, Francisco Hernández Escobar, MD, David Pérez Alonso, MD, PhD, and
}

Luis López Rivero, MD, PhD, Las Palmas de Gran Canaria, Spain

\footnotetext{
From the Department of Thoracic Surgery, Hospitalario Universitario Insular de Gran Canaria, Las Palmas de Gran Canaria, Spain.

Disclosures: Authors have nothing to disclose with regard to commercial support.

Received for publication July 7, 2017; revisions received Aug 4, 2017; accepted for publication Aug 27, 2017; available ahead of print Oct 3, 2017

Address for reprints: Jose Ramón Cano García, MD, H.U. Insular de Gran Canaria, Avda Marítima del Sur, s/n CP 35016, Las Palmas de Gran Canaria, Spain (E-mail: joseravich@gmail.com).

J Thorac Cardiovasc Surg 2018;155:e59-60

$0022-5223 / \$ 36.00$

Copyright (C) 2017 by The American Association for Thoracic Surgery

https://doi.org/10.1016/j.jtcvs.2017.08.118
}

Chest wall reconstructions, which are mainly needed after surgical treatment of oncologic disease or trauma, still represent a challenge for chest surgeons. No consensus exists regarding the materials or prostheses to be used. A wide variety of methods and materials are therefore available for chest wall reconstruction, and the choice generally depends on the location and size of the defect to be covered. To this day, most prostheses provide the chest wall with the necessary hardness and stability to protect inner organs; however, they restrict normal respiratory movements because of their rigidity. Moreover, there is the problem of finding good anchor points, because the patient has to adapt to the prosthesis, and exact adjustment is usually not possible. Designing personalized prostheses with 3-dimensional (3D) technology may provide a solution to this problem. To our knowledge, we present here the first case of complete reconstruction of the anterior chest wall with a flexible, patient-tailored prosthesis designed with 3D technology, which did not restrict respiratory movements and allowed normal respiratory function.

\section{CLINICAL SUMMARY}

A female patient had undergone surgery 16 years before for bone fibrous dysplasia of the sternum. A relapse at 8 years required complete resection of the sternum and first 3 bilateral ribs, with placement of a polytetrafluoroethylene prosthesis in the mediastinum and a metal mesh. This broke, causing instability of the anterior chest wall, pain, restrictions to shoulder mobility, and paradoxic movements of the chest wall, which impaired respiration.

A new intervention was indicated to remove the mesh and implant a new prosthesis that covered the wall defect and restored normal chest anatomy and physiology. We first proceeded to perform a 3D computed tomographic reconstruction of the patient to calculate the dimensions of the bone defect to be covered. We collaborated with biomedical engineers to design a prosthesis that was perfectly adapted

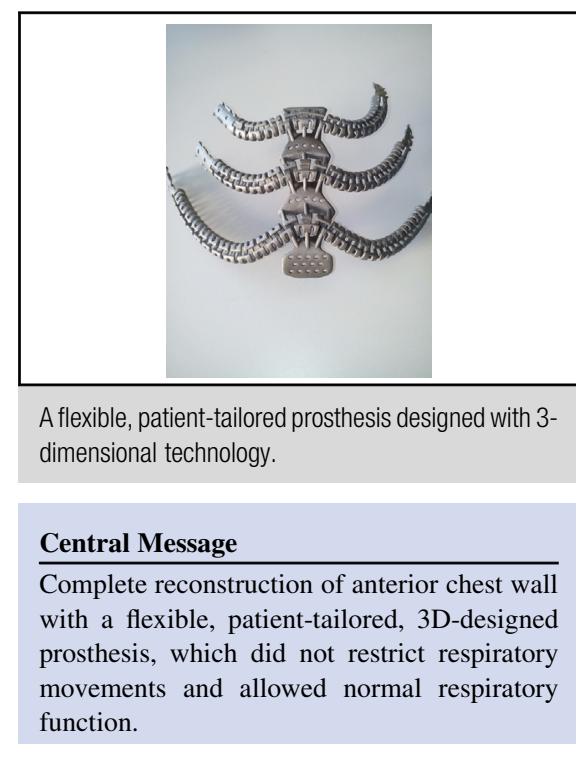

See Editorial Commentary page e61. to our patient, with $2 \mathrm{~cm}$ of lateral excess in case the resection was greater. In addition to having a small additional footprint and being an adaptable prosthesis, it simulated a sternum with its 3 ribs articulated bilaterally, a flexible system that allowed the flexing and elongation of these ribs with respiratory movements. The prosthesis was made from titanium for its lightness, hardness, and good tolerance with 3D printing. We designed the prosthesis at its ends, grooved and with holes to be able to fix both with screws and with points of wire and cerclage with braided wire because it was more comfortable. Once fixed in place, the prosthesis provided the same hardness and flexibility as a normal thorax.

The surgical intervention consisted of removing the old polytetrafluoroethylene and metal meshes, placing a new polytetrafluoroethylene mesh on the mediastinum, implanting the titanium prosthesis, and fixing it to the ribs on the chest sides with braided cerclage wire and screws. The implant was covered with the chest muscles so that it did not contact the skin.

The patient was discharged 5 days after the operation with complete remission of symptoms and normal ventilatory mechanics. Three months later, a radioscopic study of the patient's respiratory movements demonstrated that the prosthesis was moving in unison with the rest of the 




FIGURE 1. Computed tomography scan reconstruction previous to the surgery and posterior to the prosthesis implant, 1 year later.

thoracic cage, without restrictions. A year and a half after the surgery, the patient is symptom free, does not have dyspnea, and performs daily exercise. A computed tomographic scan a year later confirms the correct placement of the prosthesis (Figure 1).

\section{DISCUSSION}

Performing chest wall resection surgery entails 2 big difficulties: covering the defect-especially in extensive resection-to protect inner organs, and avoiding impairment of respiratory movements. In our case, there was also a risk associated with the fact that it was the third surgical intervention (difficulties inherent in such an intervention, such as adhesions, fibrosis, difficulty in fixing the prosthesis, and new rupture or intolerance). ${ }^{1,2} \mathrm{~A}$ prosthesis with the following characteristics was therefore necessary:

1. It had to provide hardness and stability comparable to a normal chest wall, to protect the inner organs, but without being excessively heavy because of its large size.

2. It had to be flexible enough to prevent impairing the respiratory movements.

3. It had to be adapted to our patient as much as possible, and it had to be easy to put in place, because this was a reintervention, so the patient's tissues were relatively unstructured.

Unlike a standard prosthesis, the 3-dimensional reconstruction of the patient's thorax allowed us to design a prosthesis that adjusted exactly to the bone defect that it had to replace. ${ }^{3,4}$ The titanium prosthesis has demonstrated advantages in terms of hardness, flexibility, and light weight relative to other materials used to date. The system by which the ribs were articulated to the sternum, in addition to providing the necessary flexibility, made it easier to place the prosthesis into position. Its flexibility may also help to absorb potential impact, preventing it from breakage, in a similar way to a normal thorax. Furthermore, the springlike design allowed a slight elongation of the prosthesis at the rib level, similar to the respiratory movements.

This prosthesis represents an important technologic advance relative both to older, more rigid prostheses (metal mesh, cement, bars) and to more recent prostheses, which have a certain degree of flexibility because they are based on the use of wires but still do not provide the same elongation as in physiologic chest movements. ${ }^{5}$ We would like to highlight the fact that this new-generation prosthesis type enhances respiratory activity, an outcome supported by the excellent recovery of our patient. She was discharged 5 days after the operation and was living a normal life without respiratory impairment 1.5 years afterward.

The disadvantage is that the cost is a little higher than a conventional prosthesis. This is justified, however, when it comes to reinterventions, large resections of the anterior thoracic wall, or involvement of joints.

To the best of our knowledge, this report presents the first complete and physiologic reconstruction of the anterior chest wall (sternum and 6 ribs) to use 3D technology to design and build a personalized functional prosthesis with a degree of flexibility that allowed for normal respiratory movements. There are no earlier references in the literature on this topic.

\section{References}

1. Khullar OV, Fernandez FG. Prosthetic reconstruction of the chest wall. Thorac Surg Clin. 2017;27:201-8.

2. Guillén G, García L, Marhuenda C, Pellisé F, Molino JA, Fontecha CG, et al. Thoracic wall reconstruction with bioabsorbable plates in pediatric malignant thoracic wall tumors. J Pediatr Surg. 2017;52:377-81.

3. Aranda JL, Jiménez MF, Rodríguez M, Varela G. Tridimensional titanium-printed custom-made prosthesis for sternocostal reconstruction. Eur J Cardiothorac Surg. 2015;48:e92-4.

4. Wang L, Cao T, Li X, Huang L. Three-dimensional printing titanium ribs for complex reconstruction after extensive posterolateral chest wall resection in lung cancer. J Thorac Cardiovasc Surg. 2016;152:e5-7.

5. Aragón J, Pérez Méndez I. Dynamic 3D printed titanium copy prosthesis: a novel design for large chest wall resection and reconstruction. J Thorac Dis. 2016;8: E385-9. 